\title{
Non Specific Amplification with the LAMP Technique in the Diagnosis of Tuberculosis in Sri Lankan Settings
}

\author{
K. D. Senarath ${ }^{1,2 \#, ~ R . ~ B . ~ U s g o d a a r a c h c h i ~}{ }^{1,2 \#, ~ V . ~ N a v a r a t n e ~}{ }^{1}$, A. Nagahawatte ${ }^{3}$, \\ C. D. Wijayarathna ${ }^{2}$, J. Alvitigala4, C. L. Goonasekara ${ }^{1 *}$ \\ ${ }^{1}$ Faculty of Medicine, General Sir John Kotelawala Defence University, Ratmalana, Sri Lanka \\ ${ }^{2}$ Department of Chemistry, University of Colombo, Colombo 03, Sri Lanka \\ ${ }^{3}$ Faculty of Medicine, University of Ruhuna, Galle, Sri Lanka \\ ${ }^{4}$ Central Chest Clinic and Hospital, Welisara, Sri Lanka \\ Email: ${ }^{*}$ charithalg@kdu.ac.lk
}

Received 25 August 2014; revised 28 September 2014; accepted 10 October 2014

Copyright ( 2014 by authors and Scientific Research Publishing Inc.

This work is licensed under the Creative Commons Attribution International License (CC BY). http://creativecommons.org/licenses/by/4.0/

(c) (i) Open Access

\section{Abstract}

Background: Tuberculosis (TB) remains a burden to Sri Lanka, where the incidence of the disease has been increasing over the past decade. The lack of early and accurate detection of the disease has been the main obstacle to its control. Microscopy or the culturing of mycobacteria from clinical samples is the most commonly used TB diagnostic tools in Sri Lanka. All these methods have their own limitations. Alternative diagnostic methods are therefore of high importance. Objectives: In this study, an attempt was made to validate loop mediated isothermal amplification (LAMP), which specifically amplifies a DNA sequence very rapidly at a low cost with limited resources. Methods: Crude DNA extractions of fifty culture isolates prepared from sputum samples, which were collected from patients with suspected TB extracts, were subjected to three separate LAMP assays. One assay was specific for $16 \mathrm{~S}$ ribosomal RNA (16S rRNA) gene in genus Mycobacterium, and could detect the bacteria up to the genus level. The other two contained MTB specific primers targeting rimM or gyrB gene sequences in Mycobacterium tuberculosis (MTB), which enabled detection up to the species level. The sensitivity and specificity of the LAMP assays in the identification of mycobacteria or MTB were compared to microscopy and culture techniques. Results: Forty three out of the 47 Mycobacterium cultures were Mycobacterium-positive for LAMP assays with universal primers indicating a sensitivity of $\mathbf{9 2 \%}$ in identifying Mycobacterium genus. However, thirteen out of 14 culture negatives were also positive with LAMP assays, which showed a specificity of only $7 \%$ in identifying MTB. The results suggested a high percentage of false positives by LAMP assays as compared to culture. Based on the colour changing of ZYBR Green dye and gel electrophoresis of

\footnotetext{
"Corresponding author.

"These authors have equally contributed to conducting experiments.
}

How to cite this paper: Senarath, K.D., Usgodaarachchi, R.B., Navaratne, V., Nagahawatte, A., Wijayarathna, C.D., Alvitigala, J. and Goonasekara, C.L. (2014) Non Specific Amplification with the LAMP Technique in the Diagnosis of Tuberculosis in Sri Lankan Settings. Journal of Tuberculosis Research, 2, 168-172. http://dx.doi.org/10.4236/jtr.2014.24021 
the LAMP-amplified product, the detection of a non-specific amplification, even in the absence of target DNA, was recurrently observed. The result was the same even after following strict safety operations and laboratory practices to avoid the possibility of a cross-over contamination of MTB. Interestingly, this nonspecific DNA amplicon did not respond to digestion with BsaI restriction enzyme, suggesting that the false positives are not due to the presence of MTB. Conclusion: Under the tested conditions, the specificity of the LAMP method to identify MTB is low as compared to culture technique. Further investigations into optimizing the LAMP assay technique are required before it can be used, in its simple form, to diagnose TB in local clinical settings.

\section{Keywords}

Tuberculosis, LAMP Assay, Nonspecific Amplification

\section{Introduction}

Tuberculosis (TB) remains a significant health problem in the world, not only in "high-burden" countries, but also in Sri Lanka [1]. The Ministry of Healthcare and Nutrition, Sri Lanka has launched a National Program for Tuberculosis Control and Chest Diseases (NPTCCD), and according to NPTCCD data, about 9000 new cases of TB are notified every year, showing that TB continues to be a major public health concern in the country.

The main TB diagnostic methods currently practiced in the region are sputum smear, culture, and PCR, where culture and PCR facilities are being available only at very limited places. However, these methods have their own limitations. Microscopy of Mycobacterium in clinical samples and the subsequent culturing of bacteria are labor-intensive and lengthy, needing special laboratory facilities. Microscopy alone can diagnose the disease only up to the generic level of the bacterium. Studies have shown that only 50 to $80 \%$ of patients with pulmonary tuberculosis become positive for microscopy [2]. Therefore, inaccurate reporting and misdiagnosing can occur. Molecular level methods based on PCR technology are also available for more specific diagnosis [3]; however, complexity of the procedure and the requirement for sophisticated and costly instruments and trained technical support makes it an expensive tool unsuitable for resource limited settings found in developing countries like Sri Lanka. Thus a critical obstacle for proper TB control has been the lack of early and accurate detection of the disease. The establishment of new low cost, less sophisticated, testing methods with high sensitivity and specificity has therefore been a timely need.

A relatively new, simple, molecular level method called loop mediated isothermal amplification (LAMP) is a promising tool for rapid diagnosis of infectious diseases, including TB, with minimal infrastructure requirements and technical training [4]-[7]. The method is based on amplifying DNA into a zigzag chain-like structure with several inverted repeats of the target connected by loops. Extremely high amounts of amplified product enable visual detection of the test using a turbidity change or fluorescence. Unlike PCR, LAMP is performed at a single constant temperature with no thermal cycling and can be completed within $30-120$ minutes. The specificity of the test is high as the reaction uses six primers that are specifically designed to recognize six distinct regions of the target bacterial DNA [6]. This technique does not require sophisticated equipment or special laboratory facilities; therefore, the associated cost is also low. The simplicity of the technique makes it suitable for point-ofcare testing at hospitals and primary care facilities, which would greatly help improve TB control in the country. This study therefore was an attempt to validate the LAMP technique for TB diagnosis at Sri Lankan laboratory settings.

\section{Methodology}

\subsection{Study Design}

Culture isolates (50 samples) prepared from sputum, which were collected from patients with suspected TB, at the Central hest clinic and hospital, Welisara, Sri Lanka were subjected to the LAMP assay. These cultures were pre-identified as MTB or MOTT (Mycobacterium other than MTB) samples using conventional microscopy and culture techniques, and those results were compared to LAMP method. Based on microscopy and culture results, forty seven of these cultures were identified as mycobacteria, twenty three cultures as Mycobacterium tubercu- 
losis (MTB), and seventeen cultures as MOTT.

Three separate LAMP reactions were carried out. One assay is to identify Mycobacterium genus using Mycobacterium universal (Muniv) primers [8]. The other two assays used MTB specific primers targeting rimM gene sequence [9] or $\operatorname{gyr} B$ gene sequence [8], which enabled detection up to the species level. A culture of standard MTB strain, H37Rv, was used as a positive control. Two negative controls were set up in which either sample or the enzyme was replaced by distilled water. The experimenter was blind to the identity of the sample, as confirmed by microscopy and culture methods.

\subsection{LAMP Assay}

The cultures were lysed by incubating in a boiling water bath, for $10 \mathrm{~min}$, and the supernatant $(11 \mu \mathrm{l})$ after centrifugation of the lysate was used for LAMP reaction as previously described [9]. Initially, amplification was carried out at constant $65^{\circ} \mathrm{C}$ temperature for $60 \mathrm{~min}$ according to previously published optimum assay conditions [9]. The reaction was terminated by heating in a boiling water bath for 2 min to inactivate the polymerase. Detection was carried out by adding $1 \mu 1$ of $1 / 10$ diluted original SYBR green I DNA stain to the tube after the amplification and observing the colour of the solution by naked eye or under UV light (the reaction mixture becomes orange with no DNA amplification whereas turns green with DNA amplification), hence Calcein and Manganese chloride was not included in the reaction mixture.

\section{Results and Discussion}

Forty three out of the 47 Mycobacterium cultures were Mycobacterium-positive for LAMP assay with universal primers indicating a sensitivity of $92 \%$ in identifying Mycobacterium genus. Since there were only three non Mycobacterium cultures, a proper indication about the specificity could not be obtained from this assay. When the LAMP assay was repeated with MTB specific primers, it was a surprising observation that most of the cultures (where MTB and MOTT samples were approximately in equal numbers) being MTB positive. Thirteen out of 14 culture negatives were also positive with LAMP assay, which showed a specificity of only $7 \%$. This indicated a high percentage of false positives by LAMP assay as compared to culture, therefore the ability of the method to identify a negative sample as a MOTT culture were very low. Surprisingly, in most occasions, the negative control, which contained only distilled water but no target DNA also turned green colour upon addition of SYBR green I indicating DNA amplification (Figure 1). Therefore, the false positives could not be simply attributed to a smaller sample size. This nonspecific amplification, in the absence of a target DNA, was evident with all three sets of primers used in the study. Only the negative control where Bst polymerase was omitted from the reaction remained orange (Figure 1).

LAMP amplified products were then analyzed using $0.8 \%$ agarose gel electrophoresis. Products from the negative controls further showed a banding pattern with an intensity similar to the positive sample, indicating that the non-specific amplification is as high as the target DNA amplification. It also seemed very unusual for a simple reagent/carry-over contamination, to yield an amount of amplicons at a similar level to the amplification in the positive sample. However, assuming that the false positives are due to a reagent/carry-over contamination of a positive sample/amplicon, all the reagents were freshly prepared taking strict precautions to avoid any possible carry-over contamination. Separate working areas and pipettes, with filtered pipette tips, were used for each step of the reaction procedure; reagent preparation, amplification and amplicon detection. All the stock and the working solutions were prepared in a laminar flow using PCR grade water. The pipettes were disinfected for several hours under UV light. The bench tops and pipettes were thoroughly disinfected with $70 \%$ ethanol, before and after setting up the reaction and detection. None of these safety measures reduced the non-specific amplification in the negative control. Assuming that the problem is with the current laboratory setup, the assay was tried out at two other laboratories, one being a brand new molecular biology laboratory. The results were similar as previous. Having confidently undertaken all the precautions and requirements to avoid any possible contamination, and having followed the standard protocol exactly as it has previously been reported, the observed nonspecific amplification was not eliminated nor reduced.

We investigated what is being amplified in the negative controls, in the absence of a target DNA, whether it is due to a possible contamination of MTB circulating in the air, which would be unavoidable unless highly sophisticated sterile laboratory conditions are maintained, or due to a primer self amplification. LAMP amplified products were subjected to digestion with the restriction enzyme BsaI [8], which contains its recognition site 
within the region being amplified by the gyrB primers, but exclusive to primers. LAMP amplicons in the positive and negative samples were purified, re-amplified with PCR using the two gyrB loop primers and digested $B s a I$. As visualized in a $0.8 \%$ agarose gel, only the amplicon in the positive reaction showed the presence of digestion (Figure 2), indicating the presence of BsaI cutting site only in the positive sample. Electrophoresis pattern of the digested amplicon from the negative control was the same as its undigested amplicon. When LAMP amplified products were directly subjected to digestion, without reamplifying with PCR, the digested positive amplicon gave a band close to the $100 \mathrm{bp}$ marker, corresponding to the predicted sizes of, $88 \& 89 \mathrm{bp}$ [8], of the digested product (data not shown). Restriction digestion of the LAMP amplified products suggests that even though some amplification is evident in the negative controls/MOTT samples they are not MTB. Therefore, it shows that the non-specific amplification in the negative reactions may not simply be due to a contamination of positive sample/amplicon.

We further tried running the LAMP assay differently to the standard protocol, by varying the parameters, such as incubation/reaction time (for 30, 45, 60, $120 \mathrm{~min}$ ), enzyme concentration (at $4 \mathrm{U}, 8 \mathrm{U}$ ) and assay temperature (at $63^{\circ} \mathrm{C}, 65^{\circ} \mathrm{C}, 66^{\circ} \mathrm{C}, 67^{\circ} \mathrm{C}, 68^{\circ} \mathrm{C}, 70^{\circ} \mathrm{C}$ ), with the anticipation of optimizing the assay conditions that might eliminate the non-specific amplification. However yet we did not succeed in obtaining a true negative control.

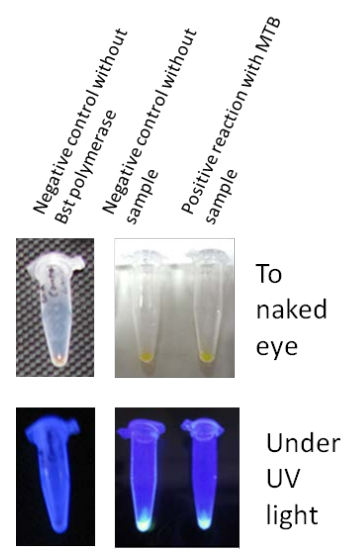

Figure 1. Three LAMP reaction mixtures, with MTB specific primers targeting rim $M$ gene sequence, were subjected to amplification for $60 \mathrm{~min}$ at $65^{\circ} \mathrm{C}$. One is the positive reaction with a culture positive MTB sample and the other two are negative controls, either without the sample DNA or without Bst polymerase. DNA amplification was detected by visualizing the colour of the final reaction mix after addition of ZYBR green to naked eye and under the UV light.

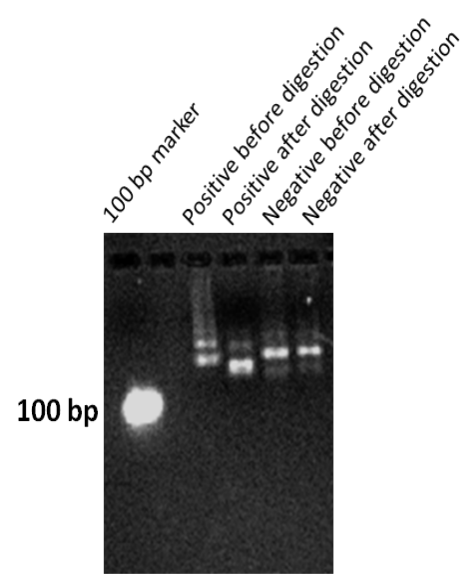

Figure 2. LAMP amplified products in the presence or absence of a MTB postive sample were re-amplified by PCR technique and subjected to disgestion with BsaI restriction enzyme. PCR amplified DNA before and after the digestion were electrophoresed on a $0.8 \%$ agarose gel. 
Surprisingly, this kind of nonspecific amplification has neither been recorded in other studies where LAMP has been validated to diagnose TB nor been recorded as a common flaw that could occur with LAMP technique [4][9]. Considering the area of the target region covered by the six primers used in the amplification of MTB by LAMP assay, which is about $95 \%$, we suspect that this nonspecific amplification could be due to any kind of self amplification of the primers.

\section{Conclusion}

Under the current circumstances, the implementation of LAMP technique as a diagnostic tool for TB in a clinical setup with less sophisticated laboratory settings needs more investigation. LAMP assay may be used if the detection is be carried out by restriction digestion. However, the simple visual detection being one of the main advantages of the LAMP assay, particularly in resource limited settings, further assay parameters, such as testing a new set of primers, is worth being studied in the future where the observed non-specific amplification might be eliminated.

\section{Acknowledgements}

KDU Research Grants from General Sir John Kotelawala Defence University is acknowledged for providing financial assistance and laboratory facilities for this study. Central hest clinic and hospital, Welisara, Sri Lanka is acknowledged for providing samples. The Department of Microbiology, Faculty of Medicine, University of Ruhuna, and the Department of Chemistry, University of Colombo are acknowledged for providing laboratory facilities to conduct the assays at different study locations.

\section{References}

[1] World Health Organization (2014) Communicable Diseases, TB in South East Asia. http://www.who.int/gho/tb/en/

[2] Dunlap, N.E., Bass, J., Fujiwara, P., Hopewell, P., Horseburgh, P.R., Salfinger, M. and Simone, P.M. (2000) Diagnostic Standards and Classification of Tuberculosis in Adults and Children. American Journal of Respiratory and Critical Care Medicine, 161, 1376-1395. http://dx.doi.org/10.1164/ajrccm.161.4.16141

[3] Ling, D.I., Flores, L.L., Riley, L.W. and Pai, M. (2008) Commercial Nucleic-Acid Amplification Tests for Diagnosis of Pulmonary Tuberculosis in Respiratory Specimens: Meta-Analysis and Meta-Regression. PLoS One, 3, e1536.

[4] Boehme, C.C., Nabeta, P., Henostroza, G., Raqib, R., Rahim, Z., Gerhardt, M., Sanga, E., Hoelscher, M., Notomi, T., Hase, T. and Perkins, M.D. (2007) Operational Feasibility of Using Loop-Mediated Isothermal Amplification for Diagnosis of Pulmonary Tuberculosis in Microscopy Centers of Developing Countries. Journal of Clinical Microbiology, 45, 1936-1940. http://dx.doi.org/10.1128/JCM.02352-06

[5] Geojith, G., Dhanasekaran, S., Chandran, S.P. and Kenneth, J. (2010) Efficacy of Loop Mediated Isothermal Amplification (LAMP) Assay for the Laboratory Identification of Mycobacterium Tuberculosis Isolates in a Resource Limited Setting. Journal of Microbiological Methods, 165, 211-220.

[6] Tomita, N., Mori, Y., Kanda, H. and Notomi, T. (2008) Loop-Mediated Isothermal Amplification (LAMP) of Gene Sequences and Simple Visual Detection of Products. Nature Protocols, 3, 877-882.

http://dx.doi.org/10.1038/nprot.2008.57

[7] Poon, L.L., Wong, B.W., Ma, E.H., Chan, K.H., Chow, L.M., Abeyewickreme, W., Tangpukdee, N., Yuen, K.Y., Guan, Y., Looareesuwan, S. and Peiris, J.S. (2006) Sensitive and Inexpensive Molecular Test for Falciparum Malaria: Detecting Plasmodium Falciparum DNA Directly from Heat-Treated Blood by Loop-Mediated Isothermal Amplification. Clinical Chemistry, 52, 303-306. http://dx.doi.org/10.1373/clinchem.2005.057901

[8] Iwamoto, T., Sonobe, T. and Hayashi, K. (2003) Loop-Mediated Isothermal Amplification for Direct Detection of Mycobacterium Tuberculosis Complex, M. avium, and M. intracellulare in Sputum Samples. Journal of Clinical Microbiology, 41, 2616-2622. http://dx.doi.org/10.1128/JCM.41.6.2616-2622.2003

[9] Zhu, R.Y., Zhang, K.X., Zhao, M.Q., Liu, Y.H., Xu, Y.Y., Ju, C.M., Li, B. and Chen, J.D. (2009) Use of Visual Loop-Mediated Isotheral Amplification of rimM Sequence for Rapid Detection of Mycobacterium tuberculosis and Mycobacterium bovis. Journal of Microbiological Methods, 78, 339-343. http://dx.doi.org/10.1016/j.mimet.2009.07.006 
Scientific Research Publishing (SCIRP) is one of the largest Open Access journal publishers. It is currently publishing more than 200 open access, online, peer-reviewed journals covering a wide range of academic disciplines. SCIRP serves the worldwide academic communities and contributes to the progress and application of science with its publication.

Other selected journals from SCIRP are listed as below. Submit your manuscript to us via either submit@scirp.org or Online Submission Portal.
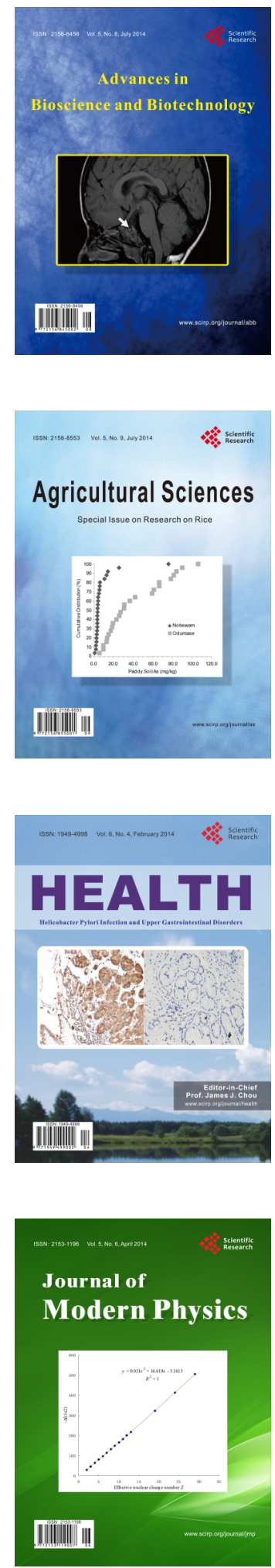
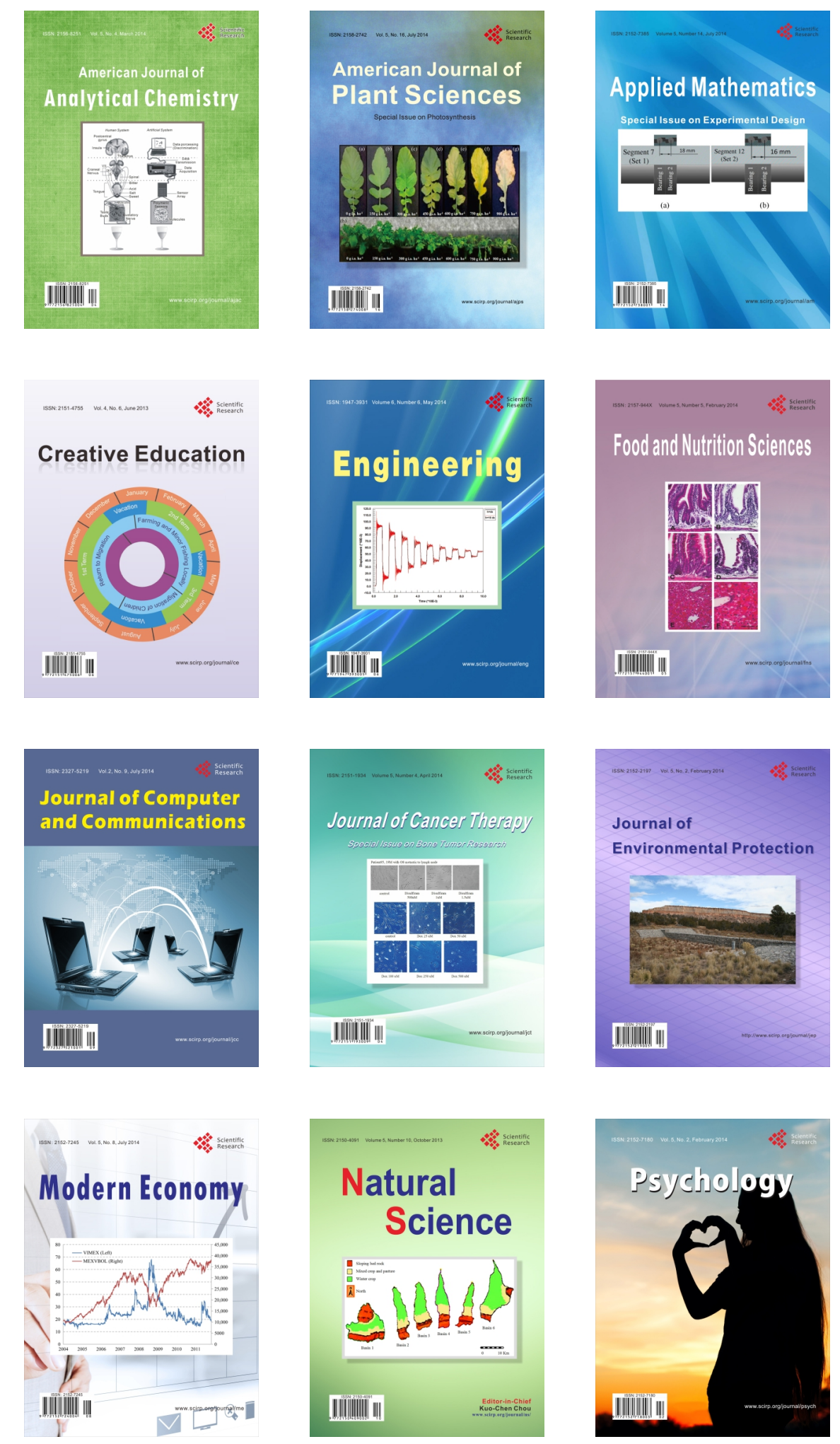